\title{
Possible roles of platelets in liver transplantation: regeneration and cancer recurrence
}

\section{Sangbin Han}

Department of Anesthesiology and Pain Medicine, Samsung Medical Center, Sungkyunkwan University School of Medicine, Seoul, Korea

When tissue injury results in breakage, platelets are not only involved in plug formation and wound sealing, but they also play an important role throughout the tissue recovery process. Specifically, platelets accumulate at the site of injury and release a large number of biologically active mediators at injury sites, which initiate or modulate damaged tissue regeneration. Moreover, extensive experimental evidence has elucidated the involvement of platelets in tumor growth and metastasis. As such, this mini-review aimed to highlight the relatively lesser known functions of platelets.

Keywords: Hepatocellular carcinoma; Liver regeneration; Living donors; Metastasis; Recurrence; Transfusion-related acute lung injury.

\section{INTRODUCTION}

Platelets have long been considered to play an important role in plug formation and wound sealing following tissue breakage secondary to injury. However, recent research has further suggested that platelets play a critical role throughout the tissue repair process aside from plug formation, with extensive experimental evidence elucidating their involvement in cancer metastasis [1-3]. Platelets are versatile cells involved in many biological processes, such as stimulating inflammation, alerting immune cells, attacking microbial pathogens, and shaping the vascular system [1]. On the other hand, platelets are also known to play certain roles in disease conditions, such as in the development of stroke, heart attack, and rheumatoid arthritis, among others [1]. Therefore, among these various functions, the current mini review focused on the role of platelets in tissue regeneration and metastasis.

\section{PLATELETS IN POST-TRANSPLANT LIVER REGENERATION}

Platelets primarily accumulate at the site of tissue damage, wherein vascular defects are sealed as part of the coagulation cascade. Furthermore, they release a large number of biologically active mediators at these injury sites, initiating or modulating immune responses, inflammation, angiogenesis, and damaged tissue regeneration $[1,4,5]$. Notably, these mediators are primarily stored in $\alpha$-granules of resting platelets and include a number of growth factors, such as hepatocyte growth factor (HGF), vascular endothelial growth factor, and insulin-like growth factor-1, all of which play important roles in liver repair and regeneration [6-10].

Liver regeneration is defined as the process of adjusting the liver mass size to meet functional demands, starting immediately as needed and resolving in only a few days or weeks $[6,7]$. Among the different types of liver cells, hepatocytes were found to be the first to proliferate and trigger various signals to promote the regeneration of other liver cells,

This is an Open Access article distributed under the terms of the Creative Commons Attribution Non-Commercial License (http://creativecommons.org/licenses/by-nc/4.0) which permits unrestricted non-commercial use, distribution, and reproduction in any medium, provided the original work is properly cited.

Copyright (C) the Korean Society of Anesthesiologists, 2021 
in which platelet-released mediators were found as the most important contributors for the initiation of hepatocyte proliferation [6-10]. In particular, previous studies performed in rats showed that plasma HGF concentration rose immediately and liver HGF receptor was activated within $30 \mathrm{~min}$ after hepatectomy, whereas the intrahepatic HGF reserve was rapidly consumed during the first three hours after hepatectomy $[11,12]$. Additionally, other platelet-released mediators, such as serotonin, were also known to be important in initiating mitogenic signaling and triggering hepatocyte proliferation $[9,10]$. Given this evidence, it could be deduced that the presence of a sufficient number of functioning platelets to deliver and release mediators to the graft immediately after liver graft reperfusion is crucial in liver transplantation, particularly in living donor liver transplantation requiring quantitative liver regeneration [13]. The positive association between intraoperative platelet transfusion and graft regeneration in our research team's recent study also supports this hypothesis $[14,15]$. To date and to the best of our knowledge, this study was the first to identify the importance of intraoperative platelet transfusion during living donor liver transplantation [14]. Particularly, our results have clinical relevance since intraoperative platelet transfusion was clearly reported to be beneficial for graft regeneration in recipients with an increased risk of liver failure, such as those with macrosteatotic or small-for-size grafts. In contrast to our study showing no negative intraoperative platelet transfusion effects, two previous studies performed in a transplant center demonstrated negative impacts of intraoperative platelet transfusion on post-transplant mortality $[16,17]$. Putative reasons for these discrepant outcomes were thought to be attributable to the differences in graft type (living donor vs. deceased donor), platelet process (leukoreduction and irradiation) $[18,19]$, transfusion indication (socalled rescue strategy: platelets were never indicated solely based on platelet counts and were always given after excessive blood loss occurred) [14], and hemostatic agent (routine aprotinin use) [20]. Apart from platelet transfusion, we also identified the importance of immediate post-reperfusion platelet counts measured during the reperfusion phase of living donor liver transplantation, which was consistent with a recent study of patients undergoing hepatectomy, suggesting that immediate postoperative platelet count was a predictor of liver regeneration [21]. Studies have shown that platelet counts during liver transplantation tend to decrease considerably according to blood loss (progressive coagulopathy and surgical bleeding) and hemodilution (fluid infusion and transfusion). In this regard, transplant teams should make efforts to maintain adequate platelet count levels during the critical period when diverse signals trigger liver regeneration. Moreover, these findings were consistent with a previous study in rats demonstrating that platelet-rich plasma infusion immediately after $70 \%$ hepatectomy promoted mitogenic signaling pathways for the first $4 \mathrm{~h}$ postoperatively, which was connected to improved early hepatocyte proliferation and liver mass recovery [22]. The authors of that study also suggested that platelet administration might be an innovative therapeutic strategy for liver regeneration in small-for-size liver transplantations.

The liver's remarkable ability to regenerate has allowed the possibility for living-donor liver transplantation to become a reality; however, this procedure has also been found to involve the potential risk of liver failure resulting from insufficient graft regeneration. Therefore, liver regeneration is a key element in the success of living donor liver transplantation. Particularly, liver regeneration is orchestrated by the interplay between various cells and mediators, of which platelet-derived mediators are known to play important roles. Notably, a landmark study by Lesurtel et al. [9] and certain subsequent studies newly discovered that platelet-derived serotonin mediated liver regeneration [8-10,15], and that triggering regeneration pathway signals occurred immediately after hepatic tissue injury, such as functional mass decrease or ischemia-reperfusion injury. More specifically, regeneration of a reduced-size partial liver graft was initiated immediately after graft reperfusion during living donor liver transplantation, and thus the intraoperative post-reperfusion period was considered to be highly critical. In this regard, our recent research showed that intraoperative platelet counts during the post-reperfusion phase and platelet transfusion were significantly associated with the degree of early graft regeneration following living donor liver transplantation [14]. Taken together, we hypothesized that intraoperative platelet counts and platelet transfusion may affect graft regeneration in relation to the serotonin pathway. Given that, we then evaluated the association between intraoperative platelet counts and serum serotonin levels, as well as the effect of intraoperative platelet transfusion on serum serotonin levels in living donor liver transplantation. Indeed, this was the first study to describe intraoperative changes in serum serotonin levels during living donor liver transplantation, measured using liquid chromatography with tandem mass spectrometry, which has been established highly reliable method. On observation, platelet counts and serotonin 
levels moved in the same direction throughout the surgical procedure. An immediate increase during the first hour after graft reperfusion followed by a gradual decrease was also observed, possibly reflecting initial systemic platelet extraction and subsequent hepatic platelet exhaustion [23]. On analysis, platelet counts and serotonin levels showed significant linear correlations. In healthy individuals, more than $95 \%$ of serotonin exists in the platelet, resulting in almost similar serum serotonin and intraplatelet serotonin levels $[24,25]$. In our findings, this similarity in the intraoperative movement of platelet counts and serum serotonin levels combined with the linear correlation between them indicated that most serotonins existed in platelets and were delivered by them in liver transplant recipients, resulting to platelet counts and serum serotonin levels that were physiologically similar to healthy individuals [10]. Therefore, our findings supported the hypothesis that the association between platelet count and early graft regeneration after living donor liver transplantation may be mediated by serotonin [14]. In addition, the platelet transfusion dose for cirrhotic patients was unknown despite its clinical importance [26]. We found that 1 unit of apheresis platelets, which corresponds to 6 units of whole blood platelets, increased platelet counts by $30 \times 10^{9} / \mathrm{L}$, which was the amount expected in healthy persons. This immediate increase in serotonin levels following platelet transfusion also supported the hypothesis that the association between platelet transfusion and early graft regeneration after living donor liver transplantation may be mediated by serotonin. However, it should be noted that platelet-mediated liver graft regeneration was mediated by other various cytokines and growth hormones aside from serotonin $[6-8,10]$. Thus, further studies are warranted to distinguish the respective roles of serotonin from other platelet-derived molecules, such as HGF, vascular endothelial growth factor, and insulin-like growth factor-1.

\section{PLATELETS IN POST-TRANSPLANT METASTASIS AND TUMOR RECURRENCE}

Liver transplantation has been a well-established therapeutic option for hepatocellular carcinoma (HCC) treatment, addressing both underlying liver diseases, which is considered a premalignant lesion, and a tumor burden. Despite this, many recipients experience tumor recurrence, wherein recurrent HCC becomes a major cause of death for these patients. As mentioned previously, platelets are the primary cells for hemostasis and tissue repair, but extensive experimental evidence has also elucidated their involvement in tumor growth and metastasis [1-3]. More specifically, clinical evidence has demonstrated an association between higher platelet counts with both shorter survival time and increased recurrence after treatment in various solid tumors [27]. In HCCs, a retrospective analysis using a large database of biopsy-proven HCC patients reported a positive correlation between platelet count and tumor size/total tumor mass [28]. Another retrospective cohort study demonstrated that pretreatment platelet count was positively associated with the probability of HCC recurrence after curative treatment [29]. These findings, as well as those from other previous studies, suggested that the relationship between platelets and tumors was not a simple epiphenomenon resulting from tumor-induced thrombopoiesis, but rather a direct contribution of platelets to multiple steps in cancer progression, including tumor cell angiogenesis, protection from immune processes, extravasation, and arrest within the vasculature $[2,27,30,31]$. In fact, a previous study in hepatitis B transgenic mice clearly demonstrated that antiplatelet treatment reduced intrahepatic inflammatory responses and HCC development with improved long-term survival [32]. Thus, our research team hypothesized that the risk of HCC recurrence after living donor liver transplantation was affected by platelets [33]. Given that, we then evaluated the relationship between preoperative platelet count and post-transplant HCC recurrence, demonstrating the independent association between them. Recurrence risk was greater with higher platelet counts after adjustment for confounders, such as tumor biology and liver disease severity in both the pre- and post-matching cohorts.

Accordingly, the risk of post-transplant HCC-related deaths was also greater with higher platelet counts. Notably, the influence of platelets was consistent regardless of tumor aggressiveness, which was an important finding since a more aggressive tumor biology due to higher platelet counts could be considered a bias. Moreover, the incorporation of platelet count into the Milan criteria significantly increased its predictive power for estimating recurrence, and no inflammation-based scores, including platelet-to-lymphocyte ratio, were superior to preoperative platelet count, which was in agreement with a previous study [34]. Particularly, the platelet-to-lymphocyte ratio was analyzed as significant, despite the insignificance of neutrophil or lymphocyte counts as well as the neutrophil-to-lymphocyte ratio, supporting the importance of platelets per se. In contrast to the surrogate markers, platelets are effector cells directly interacting with cancer cells 
in the metastatic cascade [1-3,27,30,31], in which their association with tumor recurrence appears to be robust and independent in liver transplant recipients, whose systemic inflammation status and platelet counts are both affected by liver disease. Taken together, the results from our study suggested a potentially important role of platelets in HCC metastasis after living donor liver transplantation, as well as demonstrated the following important clinical implications [32,35,36]. First, the decision-making processes of HCC staging and management largely depended on estimated recurrence probability [37]. Our study indicated that HCC management guidelines could be refined by considering platelet counts. Second, the data supported the feasibility of platelet modulation therapies and its selective implication based on platelet count, which has a spectrum of feasible therapies, including cyclooxygenase, protease-activated receptor, and integrin GPIIb/IIIa inhibitors [2,32,35,36,38,39]. Until more scientific data verifying their safety is accumulated, HCC patients with mild thrombocytopenia, in particular, are likely better candidates for these therapies, since such patients can have less bleeding diathesis and greater metastasis risks. Furthermore, the stable late post-transplant phase might provide another time window for targeting latent metastasis, which has a lesser concern on bleeding complications or graft regeneration $[13,14]$. In that regard, therapies that can differentiate the platelet-cancer loop from coagulation or tissue regeneration pathways represent opportunities to prevent metastasis with minimum related complications. Third, the study suggested that restrictive perioperative platelet transfusion policies might have another advantage from the viewpoint of metastasis prevention [40].

HCC recurrence arises from tumor cells present in the circulation or in micrometastatic colonies following primary tumor removal. To metastasize successfully, disseminated tumor cells must complete multiple complex steps, consisting of survival in the blood stream, adherence to the vascular wall, subsequent extravasation and initial seeding, and lastly, the re-initiation of growth [31]. During this metastatic cascade, platelets interact with tumor cells at every step in circulation, as well as at the site of distant metastases, while directly enhancing invasive potential or indirectly modulating the microenvironment $[2,27,41,42]$. For example, platelet-tumor cell cross-linking forms a shield around tumor cells and protects them from mechanical destruction by hemodynamic shear forces and immunologic lysis by natural killer cells. Additionally, they also promote tumor cell arrest to the secondary site through rolling and tethering to the vessel wall. Regarding cellular motility, platelet-derived mol- ecules promote extravasation by increasing endothelial permeability and inducing morphological changes from epithelial to mesenchymal transition. Moreover, platelets are known to release proangiogenic growth factors, such as the vascular endothelial, platelet-derived, fibroblast, insulin-like, and transforming growth factors $[2,10]$. Although extensive experimental research has outlined the platelet-cancer loop, clinical studies also support the importance of platelets by demonstrating an association between platelet count and poor prognosis in cancers of various organs, including the liver $[27,29,43]$. Interestingly, sorafenib, the first systemic drug to be approved for advanced HCC management, was found to suppresses HCC progression by inhibiting cellular signaling mediated by platelet-derived growth factors, such as the vascular endothelial and platelet-derived growth factors [44].

Furthermore, recent studies have consistently demonstrated that early post-transplant platelet count was mainly determined by preoperative platelet count [45]. Thus, it could be deduced that patients with higher preoperative platelet counts continue to experience greater platelet-tumor cell interactions and consequently a more favorable metastasis microenvironment during early post-transplantation when surgery-induced stress, inflammation, and immunomodulation provide a vulnerable time window. Evidence that circulating tumor cells require only a few hours to days to complete the metastatic cascade further supports the relevance of preoperative platelet count [31]. Additionally, some tumor cells may also remain dormant at the secondary site for a long period, thus initiating growth at a much later time and resulting in a metastasis that occurs over an extended duration [31]. Since post-transplant platelet counts significantly correlated with preoperative values even months or years after transplantation, recipients with higher preoperative platelet counts might have a greater chance of experiencing HCC cell metastasis in much later periods.

Platelet counts in HCC patients are determined by parenchymal liver disease severity and HCC aggresiveness [46]. More specifically, platelet production is decreased mainly due to impaired thrombopoietin synthesis in the diseased liver, whereas platelet destruction is increased due to cirrhosis-related hypersplenism and platelet-associated antibodies. Moreover, systemic inflammation increases platelet consumption and hyperdynamic circulation dilutes platelet concentration [47]. In contrast, solid tumors enhance hepatic thrombopoietin synthesis by releasing thrombopoietic cy- 
tokines, wherein more aggressive tumors induce a greater degree of thrombocytosis $[27,48]$. Accordingly, parenchymal liver disease status and tumor biology are significantly different according to preoperative platelet counts. Since some determinants of platelet count are also contributors to post-transplant outcomes, the success of the study evaluating the relationship of platelets and post-transplant metastasis mainly depends on determining whether the platelet count is an epiphenomenon. First, there is the possibility of recurrence risk being overestimated in patients with higher platelet counts with a more aggressive tumor biology. Second, there is the possibility of recurrence risk being underestimated in patients with lower platelet counts, since HCC-unrelated death was reported to be greater in this group [33]. Therefore, future studies evaluating the relationship between perioperative platelets counts or platelet transfusion, and post-transplant HCC metastasis or recurrence, should be considered with regards to their potential biasing effects as in our research [33].

\section{CONCLUSION}

Liver transplant physicians who perform perioperative platelet transfusion should be aware that platelets are not only cells involved in decreasing the amount of medical bleeding, but they are also in charge of liver regeneration and post-transplant HCC metastasis. The two relatively novel concepts are now being actively studied in various populations, including liver transplant recipients, as presented in this paper. As such, we would like to call attention to research evaluating the potential positive and negative roles of platelets and platelet transfusion in liver transplant recipients for the creation of future studies in this field.

\section{CONFLICTS OF INTEREST}

No potential conflict of interest relevant to this article was reported.

\section{DATA AVAILABILITY STATEMENT}

This is a review article written based on previous studies.

\section{ORCID}

Sangbin Han, https://orcid.org/0000-0002-4129-301X

\section{REFERENCES}

1. Leslie M. Cell biology. Beyond clotting: the powers of platelets. Science 2010; 328: 562-4

2. Tesfamariam B. Involvement of platelets in tumor cell metastasis. Pharmacology \& Therapeutics 2016; 157: 112-9.

3. Gay LJ, Felding-Habermann B. Contribution of platelets to tumour metastasis. Nat Rev Cancer 2011; 11: 123-34.

4. Stellos K, Kopf S, Paul A, Marquardt JU, Gawaz M, Huard J, et al. Platelets in regeneration. Semin Thromb Hemost 2010; 36: 175-84.

5. Gawaz M, Vogel S. Platelets in tissue repair: control of apoptosis and interactions with regenerative cells. Blood 2013; 122: 2550-4.

6. Michalopoulos GK, DeFrances MC. Liver regeneration. Science 1997; 276: 60-6.

7. Taub R. Liver regeneration: from myth to mechanism. Nat Rev Mol Cell Biol 2004; 5: 836-47.

8. Meyer J, Balaphas A, Fontana P, Sadoul K, Morel P, GonelleGispert C, et al. Platelets in liver regeneration. ISBT Sci Ser 2017; 12: 455-62.

9. Lesurtel M, Graf R, Aleil B, Walther DJ, Tian Y, Jochum W, et al. Platelet-derived serotonin mediates liver regeneration. Science 2006; 312: 104-7.

10. Clavien PA. Liver regeneration: a spotlight on the novel role of platelets and serotonin. Swiss Med Wkly 2008; 138: 361-70.

11. Stolz DB, Mars WM, Petersen BE, Kim TH, Michalopoulos GK. Growth factor signal transduction immediately after two-thirds partial hepatectomy in the rat. Cancer Res 1999; 59: 3954-60.

12. Pediaditakis P, Lopez-Talavera JC, Petersen B, Monga SP, Michalopoulos GK. The processing and utilization of hepatocyte growth factor/scatter factor following partial hepatectomy in the rat. Hepatology 2001; 34(4 Pt 1): 688-93.

13. Clavien PA, Petrowsky H, DeOliveira ML, Graf R. Strategies for safer liver surgery and partial liver transplantation. N Engl J Med 2007; 356: 1545-59.

14. Han S, Park HW, Song JH, Gwak MS, Lee WJ, Kim G, et al. Association between intraoperative platelet transfusion and early graft regeneration in living donor liver transplantation. Ann Surg 2016; 264: 1065-72.

15. Han S, Ko JS, Gwak MS, Kim GS. Association of platelet count and platelet transfusion with serotonin level during living donor liver transplantation: possible connection to graft regeneration. Transplant Proc 2018; 50: 1104-7.

16. de Boer MT, Christensen MC, Asmussen M, van der Hilst CS, Hendriks HG, Slooff MJ, et al. The impact of intraoperative transfusion of platelets and red blood cells on survival after liv- 
er transplantation. Anesth Analg 2008; 106: 32-44.

17. Pereboom IT, de Boer MT, Haagsma EB, Hendriks HG, Lisman T, Porte RJ. Platelet transfusion during liver transplantation is associated with increased postoperative mortality due to acute lung injury. Anesth Analg 2009; 108: 1083-91.

18. Parker BM, Irefin SA, Sabharwal V, Tetzlaff JE, Beven C, Younossi Z, et al. Leukocyte reduction during orthotopic liver transplantation and postoperative outcome: a pilot study. J Clin Anesth 2004; 16: 18-24.

19. Jiwaji Z, Nunn KP, Conway-Morris A, Simpson AJ, Wyncoll D, Rossi AG, et al. RELIEVE Trial Investigators. Leukoreduced blood transfusion does not increase circulating soluble markers of inflammation: a randomized controlled trial. Transfusion 2014; 54: 2404-11.

20. Xia VW, Steadman RH. Antifibrinolytics in orthotopic liver transplantation: current status and controversies. Liver Transpl 2005; 11: 10-8.

21. Alkozai EM, Nijsten MW, de Jong KP, de Boer MT, Peeters PM, Slooff MJ, et al. Immediate postoperative low platelet count is associated with delayed liver function recovery after partial liver resection. Ann Surg 2010; 251: 300-6.

22. Matsuo R, Nakano Y, Ohkohchi N. Platelet administration via the portal vein promotes liver regeneration in rats after $70 \%$ hepatectomy. Ann Surg 2011; 253: 759-63.

23. Meyer J, Lejmi E, Fontana P, Morel P, Gonelle-Gispert C, Bühler L. A focus on the role of platelets in liver regeneration: do platelet-endothelial cell interactions initiate the regenerative process? J Hepatol 2015; 63: 1263-71.

24. Starlinger P, Assinger A, Haegele S, Wanek D, Zikeli S, Schauer $\mathrm{D}$, et al. Evidence for serotonin as a relevant inducer of liver regeneration after liver resection in humans. Hepatology 2014; 60: $257-66$

25. Kema IP, de Vries EG, Muskiet FA. Clinical chemistry of serotonin and metabolites. J Chromatogr B Biomed Sci Appl 2000; 747: 33-48.

26. Stroncek DF, Rebulla P. Platelet transfusions. Lancet 2007; 370: 427-38.

27. Buergy D, Wenz F, Groden C, Brockmann MA. Tumor-platelet interaction in solid tumors. Int J Cancer 2012; 130: 2747-60.

28. Carr BI, Guerra V, Pancoska P. Thrombocytopenia in relation to tumor size in patients with hepatocellular carcinoma. Oncology 2012; 83: 339-45.

29. Lee CH, Lin YJ, Lin CC, Yen CL, Shen CH, Chang CJ, et al. Pretreatment platelet count early predicts extrahepatic metastasis of human hepatoma. Liver Int 2015; 35: 2327-36.

30. Bambace NM, Holmes CE. The platelet contribution to cancer progression. J Thromb Haemost 2011; 9: 237-49.
31. Labelle M, Hynes RO. The initial hours of metastasis: the importance of cooperative host-tumor cell interactions during hematogenous dissemination. Cancer Discov 2012; 2: 1091-9.

32. Sitia G, Aiolfi R, Di Lucia P, Mainetti M, Fiocchi A, Mingozzi F, et al. Antiplatelet therapy prevents hepatocellular carcinoma and improves survival in a mouse model of chronic hepatitis B. Proc Natl Acad Sci U S A 2012; 109: E2165-72.

33. Han S, Lee S, Yang JD, Leise MD, Ahn JH, Kim S, et al. Risk of posttransplant hepatocellular carcinoma recurrence is greater in recipients with higher platelet counts in living donor liver transplantation. Liver Transpl 2018; 24: 44-55.

34. Parisi I, Tsochatzis E, Wijewantha H, Rodríguez-Perálvarez M, De Luca L, Manousou P, et al. Inflammation-based scores do not predict post-transplant recurrence of hepatocellular carcinoma in patients within Milan criteria. Liver Transpl 2014; 20: 1327-35.

35. Mousa SA, Petersen LJ. Anti-cancer properties of low-molecular-weight heparin: preclinical evidence. Thromb Haemost 2009; 102: 258-67.

36. Okada H, Honda M, Campbell JS, Sakai Y, Yamashita T, Takebuchi Y, et al. Acyclic retinoid targets platelet-derived growth factor signaling in the prevention of hepatic fibrosis and hepatocellular carcinoma development. Cancer Res 2012; 72: 445971.

37. Clavien PA, Lesurtel M, Bossuyt PM, Gores GJ, Langer B, Perrier A; OLT for HCC Consensus Group. Recommendations for liver transplantation for hepatocellular carcinoma: an international consensus conference report. Lancet Oncol 2012; 13: e11-22.

38. Abiru S, Nakao K, Ichikawa T, Migita K, Shigeno M, Sakamoto M, et al. Aspirin and NS-398 inhibit hepatocyte growth factor-induced invasiveness of human hepatoma cells. Hepatology 2002; 35: 1117-24.

39. Hossain MA, Kim DH, Jang JY, Kang YJ, Yoon JH, Moon JO, et al. Aspirin induces apoptosis in vitro and inhibits tumor growth of human hepatocellular carcinoma cells in a nude mouse xenograft model. Int J Oncol 2012; 40: 1298-304.

40. Westerkamp AC, Lisman T, Porte RJ. How to minimize blood loss during liver surgery in patients with cirrhosis. HPB (Oxford) 2009; 11: 453-8.

41. Zhang T, Sun HC, Xu Y, Zhang KZ, Wang L, Qin LX, et al. Overexpression of platelet-derived growth factor receptor alpha in endothelial cells of hepatocellular carcinoma associated with high metastatic potential. Clin Cancer Res 2005; 11(24 Pt 1): 8557-63.

42. Carr BI, Cavallini A, D’Alessandro R, Refolo MG, Lippolis C, Mazzocca A, et al. Platelet extracts induce growth, migration and invasion in human hepatocellular carcinoma in vitro. BMC 
Cancer 2014; 14: 43.

43. Morimoto Y, Nouso K, Wada N, Takeuchi Y, Kinugasa H, Miyahara K, et al. Involvement of platelets in extrahepatic metastasis of hepatocellular carcinoma. Hepatol Res 2014; 44: E353-9.

44. Dhanasekaran R, Venkatesh SK, Torbenson MS, Roberts LR. Clinical implications of basic research in hepatocellular carcinoma. J Hepatol 2016; 64: 736-45.

45. Peck-Radosavljevic M, Wichlas M, Zacherl J, Stiegler G, Stohlawetz P, Fuchsjäger M, et al. Thrombopoietin induces rapid resolution of thrombocytopenia after orthotopic liver transplantation through increased platelet production. Blood 2000; 95:
795-801.

46. Pradella P, Bonetto S, Turchetto S, Uxa L, Comar C, Zorat F, et al. Platelet production and destruction in liver cirrhosis. J Hepatol 2011; 54: 894-900.

47. Han S, Lee JH, Kim G, Ko JS, Choi SJ, Kwon JH, et al. Bioreactance is not interchangeable with thermodilution for measuring cardiac output during adult liver transplantation. PLoS One 2015; 10: e0127981.

48. Stone RL, Nick AM, McNeish IA, Balkwill F, Han HD, Bottsford-Miller J, et al. Paraneoplastic thrombocytosis in ovarian cancer. N Engl J Med 2012; 366: 610-8. 